\title{
TWO-SIDED BOUNDS OF THE DISCRETIZATION ERROR FOR FINITE ELEMENTS
}

\author{
Michal KřížeK ${ }^{1}$, Hans-Goerg Roos ${ }^{2}$ And Wei Chen ${ }^{3}$
}

\begin{abstract}
We derive an optimal lower bound of the interpolation error for linear finite elements on a bounded two-dimensional domain. Using the supercloseness between the linear interpolant of the true solution of an elliptic problem and its finite element solution on uniform partitions, we further obtain two-sided a priori bounds of the discretization error by means of the interpolation error. Two-sided bounds for bilinear finite elements are given as well. Numerical tests illustrate our theoretical analysis.
\end{abstract}

Mathematics Subject Classification. 65N30.

Received July 8, 2010.

Published online April 6, 2011.

\section{INTRODUCTION}

The first known two-sided estimate goes back to the antiquity, when Archimedes estimated $\pi$ from below and above by means of inscribed and circumscribed regular polygons to the unit circle. Two-sided bounds of the energy of the weak solution of elliptic problems can be obtained by a simultaneous use of the primal and dual finite element method, see e.g. [9], p. 65, [12], p. 261. Two-sided a posteriori bounds of the discretization error for the finite element for elliptic problems are given e.g. in [7], p. 239-242, [15], p. 29. In this paper we introduce two-sided a priori bounds of the discretization error for linear and bilinear finite elements. To the authors' knowledge, such bounds were obtained for the first time.

Let $\Omega \subset \mathbb{R}^{2}$ be a bounded polygonal domain. Consider a strongly regular family $\mathcal{F}=\left\{\mathcal{T}_{h}\right\}_{h \rightarrow 0}$ of face-to-face triangulations $\mathcal{T}_{h}$ of $\bar{\Omega}$, i.e., there exists a constant $C>0$ such that for any $\mathcal{T}_{h} \in \mathcal{F}$ and any triangle $T \in \mathcal{T}_{h}$ we have

$$
\text { meas } T \geq C h^{2} \text {. }
$$

Throughout the paper the symbol $C$ (possibly with subindices) stands for a positive generic constant independent of the discretization parameter $h$, but which may be dependent on a given fixed function. The generic constant may attain different values at different occurrences.

\footnotetext{
Keywords and phrases. Lagrange finite elements, Céa's lemma, superconvergence, lower error estimates

1 Institute of Mathematics, Academy of Sciences, Žitná 25, 11567 Prague 1, Czech Republic. krizek@math.cas.cz

2 Institute of Numerical Mathematics, Technical University Dresden, Zellescher Weg 12-14, 01069 Dresden, Germany. hans-goerg.roos@tu-dresden.de

3 School of Economics, Shandong University, 27 Shanda Nanlu, Jinan 250 100, P.R. China. weichen@sdu.edu.cn
} 
Denote by $V_{h}$ the space of functions that are linear over each triangle $T \in \mathcal{T}_{h}$ and continuous over $\bar{\Omega}$. Then for the standard linear Lagrangian interpolation operator $L_{h}: C(\bar{\Omega}) \rightarrow V_{h}$ we have the well-known estimate

$$
\left\|v-L_{h} v\right\|_{1} \leq C h|v|_{2} \quad \text { as } h \rightarrow 0
$$

where $\|\cdot\|_{k}$ and $|\cdot|_{k}$ stand for the usual $H^{k}$-Sobolev norm and seminorm, respectively. It is said that approximation order of $L_{h}$ in (1.2) is optimal, i.e., it cannot be improved. calculating the norm $\left\|v-L_{h} v\right\|_{1}$ for a given quadratic function $v$. Then the norm is asymptotically bounded from below by $h$ multiplied by a positive constant.

In the next section we show that such a lower bound holds for an arbitrary nonlinear smooth function $v$, not only quadratic. It generalizes results from [2,3] for a one-dimensional problem into two dimensions. A similar result without proof is also stated in [14], Theorem 3.5. In Section 3 we shall apply the finite element method to a second order elliptic boundary value problem with variable coefficients. Using the lower bound for the interpolation error and some superconvergence property, we derive two-sided bounds of the discretization error by means of the interpolation error, namely

$$
(1-C h)\left\|u-L_{h} u\right\|_{1} \leq\left\|u-u_{h}\right\|_{1} \leq(1+C h)\left\|u-L_{h} u\right\|_{1} \quad \text { as } h \rightarrow 0,
$$

where $u_{h}$ is the finite element solution over uniform triangulations. Note that superconvergence results are usually applied to get a higher accuracy or some a posteriori error estimates or to perform mesh adaptation. Derivation of the two-sided a priori bounds of the discretization error $\left\|u-u_{h}\right\|_{1}$ is a new application of superconvergence theory.

An extension of results from Sections 2 and 3 to bilinear finite elements is given in Section 4 . Finally, in Section 5 some numerical tests are presented.

\section{LOWER BOUND OF THE INTERPOLATION ERROR FOR LINEAR ELEMENTS}

To show the main idea of the lower bound estimate of the interpolation error, we will first consider special uniform triangulations.

Theorem 2.1. Let $v \in C^{2+\varepsilon}(\bar{\Omega}) \backslash P_{1}(\bar{\Omega})$ for some $\varepsilon>0$. Then for a family of uniform triangulations consisting of isoceles right triangles there exists a constant $C>0$ depending on $v$ such that

$$
\left|v-L_{h} v\right|_{1} \geq C h \quad \text { as } h \rightarrow 0
$$

Proof. Since $v$ is not linear, it follows for the matrix Hes $v$ of second derivatives that

$$
M:=\max _{(x, y) \in \bar{\Omega}} \sum_{k, \ell=1}^{2}(\operatorname{Hes} v(x, y))_{k \ell}^{2}>0 .
$$

Thus, there exists a nonempty subdomain $\Omega_{0} \subset \Omega$ such that for any $(x, y) \in \Omega_{0}$ we have

$$
\sum_{k, \ell=1}^{2}(\operatorname{Hes} v(x, y))_{k \ell}^{2}>\frac{M}{2} .
$$


Let $K \in \mathcal{T}_{h}$ be a triangle with vertices $\left(x_{i}, y_{j}\right),\left(x_{i}+h, y_{j}\right),\left(x_{i}+h, y_{j}+h\right)$ and let $K \subset \Omega_{0}$. First, we shall compute $\left|v-L_{h} v\right|_{1, K}^{2}$ under the assumption that $v$ is a quadratic polynomial, i.e., $\partial_{11} v=a, \partial_{12} v=b$, and $\partial_{22} v=c$ are constants. In this special case we obtain

$$
\begin{aligned}
& \partial_{1}\left(v-L_{h} v\right)(x, y)=a\left(x-x_{i}-\frac{1}{2} h\right)+b\left(y-y_{j}\right) \\
& \partial_{2}\left(v-L_{h} v\right)(x, y)=b\left(x-x_{i}-h\right)+c\left(y-y_{j}-\frac{1}{2} h\right) .
\end{aligned}
$$

To see this, we set $z=\partial_{1}\left(v-L_{h} v\right)$ on $K$. Then the Taylor expansion of the linear function $z$ at the point $P=\left(x_{P}, y_{P}\right) \in K$ reads

$$
\begin{aligned}
z(x, y) & =z(P)+\partial_{1} z(P)\left(x-x_{P}\right)+\partial_{2} z(P)\left(y-y_{P}\right) \\
& =z(P)+a\left(x-x_{P}\right)+b\left(y-y_{P}\right) .
\end{aligned}
$$

Setting $P=D=\left(x_{i}+\frac{h}{2}, y_{j}\right)$, we come to

$$
z(x, y)=a\left(x-x_{i}-\frac{1}{2} h\right)+b\left(y-y_{j}\right),
$$

since the values of $\partial_{1} v$ and $\partial_{1} L_{h} v$ coincide at the midpoint $D$. The second equation of (2.3) can be derived similarly for the midpoint $E=\left(x_{i}+h, y_{j}+\frac{h}{2}\right)$, see also the identities for the interpolation error in [11].

Denote by $F=\left(x_{i}+\frac{h}{2}, y_{j}+\frac{h}{2}\right)$ the third midpoint of edges of $K$. Applying the midpoint quadrature rule

$$
\int_{K} p \mathrm{~d} K=\frac{\text { meas }}{3} K(p(D)+p(E)+p(F))
$$

which is valid for all quadratic polynomials $p$ (see [8], p. 55), we get by (2.3)

$$
\begin{aligned}
\left|v-L_{h} v\right|_{1, K}^{2} & =\int_{K}\left\{\left[\partial_{1}\left(v-L_{h} v\right)\right]^{2}+\left[\partial_{2}\left(v-L_{h} v\right)\right]^{2}\right\} \mathrm{d} K \\
& =\frac{1}{3} \frac{h^{2}}{2} \sum_{(x, y) \in\{D, E, F\}}\left\{\left[a\left(x-x_{i}-\frac{1}{2} h\right)+b\left(y-y_{j}\right)\right]^{2}+\left[b\left(x-x_{i}-h\right)+c\left(y-y_{j}-\frac{1}{2} h\right)\right]^{2}\right\} \\
& =\frac{h^{2}}{6} \cdot \frac{1}{4}\left\{(b h+c h)^{2}+(a h+b h)^{2}+b^{2} h^{2}+b^{2} h^{2}\right\} \\
& =\frac{h^{4}}{24}\left(a^{2}+2 a b+4 b^{2}+2 b c+c^{2}\right)
\end{aligned}
$$

This value is positive due to $(2.2)$.

Now let $v \in C^{2+\varepsilon}(K)$ and let $z=\partial_{1}\left(v-L_{h} v\right)$. Then by the Taylor expansion we get like in $(2.4)$ that

$$
z(x, y)=z(P)+a\left(x-x_{P}\right)+b\left(y-y_{P}\right)+o(h)
$$


where $a$ and $b$ are constant approximations of $\partial_{11} v$ and $\partial_{12} v$ on $K$, respectively. Using a similar expansion for $\partial_{2}\left(v-L_{h} v\right)$, we find by (2.6) that

$$
\left|v-L_{h} v\right|_{1, K}^{2}=\frac{h^{4}}{24}\left(a^{2}+2 a b+4 b^{2}+2 b c+c^{2}\right)+o\left(h^{4}\right) \geq C_{1} h^{4} \text { with } C_{1}>0
$$

for $h$ sufficiently small. The constant $C_{1}$ is independent of $K \subset \Omega_{0}$, but depends on $v$ due to (2.2). An analogous lower bound can be obtained for a triangle $K^{\prime} \in \mathcal{T}_{h}$ with vertices $\left(x_{i}, y_{j}\right),\left(x_{i}, y_{j}+h\right)$, and $\left(x_{i}+h, y_{j}+h\right)$.

Summing up (2.7) over all elements $K \subset \Omega_{0}$ (and similar inequalities for elements $K^{\prime} \subset \Omega_{0}$ ), we come to

$$
\left|v-L_{h} v\right|_{1, \Omega_{0}}^{2} \geq C_{2} h^{2}
$$

since the number of these elements is greater than or equal $C_{3} h^{-2}$. Hence, (2.1) holds.

Further, we will derive a lower bound similar to (2.1) for unstructured triangulations. The proof will be quite similar to that of Theorem 2.1, so we will present only the main points.

Consider an arbitrary triangle $T$ with vertices $A_{k}$ (defined as column vectors) for $k=0,1,2$ and the linear affine mapping $F_{T}: \hat{T} \rightarrow T$, where $\hat{T}$ is the reference triangle with vertices $\hat{A}_{0}=(0,0)^{\top}, \hat{A}_{1}=(1,0)^{\top}$, and $\hat{A}_{2}=(0,1)^{\top}$. Then

$$
\left(\begin{array}{l}
x \\
y
\end{array}\right)=F_{T}\left(\begin{array}{l}
\hat{x} \\
\hat{y}
\end{array}\right)=B_{T}\left(\begin{array}{l}
\hat{x} \\
\hat{y}
\end{array}\right)+b_{T}
$$

where

$$
B_{T}=\left(\begin{array}{ll}
b_{11} & b_{12} \\
b_{21} & b_{22}
\end{array}\right)=\left(A_{1}-A_{0}, A_{2}-A_{0}\right)
$$

is a nonsingular $2 \times 2$ matrix, $b_{T}=A_{0} \in \mathbb{R}^{2}$, and $F_{T}\left(\hat{A}_{k}\right)=A_{k}, k=0,1,2$.

Assume now that a given family of triangulations is strongly regular (see (1.1)). Then the Euclidean norm of every column of $B_{T}$ can be estimated as follows

$$
C_{1} h \leq\left\|A_{k}-A_{0}\right\| \leq C_{2} h \quad \text { for } k=1,2
$$

and

$$
C_{1} h^{2} \leq\left|\operatorname{det} B_{T}\right|=2 \text { meas } T \leq C_{2} h^{2} .
$$

For every $w \in C(T) \cap H^{1}(T)$ and every $(\hat{x}, \hat{y})^{\top} \in \hat{T}$ we define by $(2.9)$,

$$
\hat{w}(\hat{x}, \hat{y})=w(x, y)
$$

Thus, we have a one-to-one correspondence between $\hat{w}$ and $w$.

Theorem 2.2. Let $v \in C^{2+\varepsilon}(\bar{\Omega}) \backslash P_{1}(\bar{\Omega})$ for some $\varepsilon>0$. Then for a strongly regular family of triangulations there exists a constant $C>0$ depending on $v$ such that

$$
\left|v-L_{h} v\right|_{1} \geq C h \quad \text { as } h \rightarrow 0 .
$$

Proof. Since

the Euclidean norm of every row of

$$
B_{T}^{-1}=\frac{1}{\operatorname{det} B_{T}}\left(\begin{array}{cc}
b_{22} & -b_{12} \\
-b_{21} & b_{11}
\end{array}\right)
$$

$$
B_{T}^{-1}=\left(\begin{array}{ll}
\alpha & \gamma \\
\beta & \delta
\end{array}\right)
$$


can be estimated from below by $C h^{-1}$ due to $(2.10),(2.11)$, and (2.12), i.e.,

$$
\alpha^{2}+\gamma^{2} \geq C h^{-2}, \quad \beta^{2}+\delta^{2} \geq C h^{-2} .
$$

Set

$$
w=v-L_{h} v
$$

and assume that (2.2) holds. Using the transformation (2.9), (2.13), and (2.14), we get

$$
\begin{aligned}
& \partial_{1} w=\alpha \hat{\partial}_{1} \hat{w}+\beta \hat{\partial}_{2} \hat{w}, \\
& \partial_{2} w=\gamma \hat{\partial}_{1} \hat{w}+\delta \hat{\partial}_{2} \hat{w} .
\end{aligned}
$$

First assume that $w$ is a quadratic polynomial. Then $\hat{w}$ is also a quadratic polynomial and

$$
\widehat{\operatorname{Hes}} \hat{w}=\left(\begin{array}{cc}
\hat{a} & \hat{b} \\
\hat{b} & \hat{c}
\end{array}\right)=B_{T}^{\top}(\operatorname{Hes} w) B_{T},
$$

where

Analogously to (2.3) we obtain

$$
\text { Hes } w=\left(\begin{array}{ll}
a & b \\
b & c
\end{array}\right)
$$

$$
\begin{aligned}
& \hat{\partial}_{1} \hat{w}(\hat{x}, \hat{y})=\hat{a}\left(\hat{x}-\frac{1}{2}\right)+\hat{b} \hat{y}, \\
& \hat{\partial}_{2} \hat{w}(\hat{x}, \hat{y})=\hat{b} \hat{x}+\hat{c}\left(\hat{y}-\frac{1}{2}\right),
\end{aligned}
$$

where $\hat{a}=\hat{\partial}_{11} \hat{w}, \hat{b}=\hat{\partial}_{12} \hat{w}$, and $\hat{c}=\hat{\partial}_{22} \hat{w}$. From this, (2.16), and (2.12) we find similarly to (2.6) that (cf. [8], p. 46])

$$
\begin{aligned}
|w|_{1, T}^{2}= & \left|\operatorname{det} B_{T}\right| \int_{\hat{T}}\left(\left(\alpha \hat{\partial}_{1} \hat{w}+\beta \hat{\partial}_{2} \hat{w}\right)^{2}+\left(\gamma \hat{\partial}_{1} \hat{w}+\delta \hat{\partial}_{2} \hat{w}\right)^{2}\right) \mathrm{d} \hat{x} \mathrm{~d} \hat{y} \\
= & 2 \operatorname{meas} T \int_{\hat{T}}\left\{\left[\alpha\left(\hat{a}\left(\hat{x}-\frac{1}{2}\right)+\hat{b} \hat{y}\right)+\beta\left(\hat{b} \hat{x}+\hat{c}\left(\hat{y}-\frac{1}{2}\right)\right)\right]^{2}\right. \\
& \left.+\left[\gamma\left(\hat{a}\left(\hat{x}-\frac{1}{2}\right)+\hat{b} \hat{y}\right)+\delta\left(\hat{b} \hat{x}+\hat{c}\left(\hat{y}-\frac{1}{2}\right)\right)\right]^{2}\right\} \mathrm{d} \hat{x} \mathrm{~d} \hat{y}
\end{aligned}
$$

Applying the midpoint quadrature rule (cf. (2.5)), we arrive by (1.1) and (2.15) at

$$
\begin{aligned}
|w|_{1, T}^{2} & =\frac{1}{2} \operatorname{meas} T\left(\left(\beta^{2}+\delta^{2}\right)(\hat{b}+\hat{c})^{2}+\left(\alpha^{2}+\gamma^{2}\right)(\hat{a}+\hat{b})^{2}+\left(\alpha^{2}+\beta^{2}+\gamma^{2}+\delta^{2}\right) \hat{b}^{2}\right) \\
& \geq C h^{2}\left(\left(\beta^{2}+\delta^{2}\right)(\hat{b}+\hat{c})^{2}+\left(\alpha^{2}+\gamma^{2}\right)(\hat{a}+\hat{b})^{2}\right) \geq C^{\prime}\left((\hat{b}+\hat{c})^{2}+(\hat{a}+\hat{b})^{2}\right) .
\end{aligned}
$$

Hence, by (2.10), (2.11), and (2.17) we find similarly to (2.7) that

$$
|w|_{1, T}^{2} \geq C_{1} h^{4} \text { with } C_{1}>0
$$

for $w \in C^{2+\varepsilon}(T)$ and $h$ sufficiently small. Summing up these inequalities over all triangles $T \subset \Omega_{0}$, we get the desired lower bound. 


\section{Application of Supercloseness}

Consider a second order elliptic problem

$$
\begin{aligned}
-\nabla \cdot(\mathcal{A}(x, y) \nabla u) & =f & & \text { in } \Omega, \\
u & =0 & & \text { on } \partial \Omega,
\end{aligned}
$$

where $f \in L^{2}(\Omega)$ and $\mathcal{A}=\mathcal{A}(x, y)$ is a symmetric $2 \times 2$-matrix whose entries are Lipschitz continuous functions on $\bar{\Omega}$ and for which there exists a constant $C>0$ such that

$$
\xi^{\top} \mathcal{A}(x, y) \xi \geq C \xi^{\top} \xi \quad \forall \xi \in \mathbb{R}^{2} \quad \forall(x, y) \in \bar{\Omega} .
$$

Assume that $u \in H^{3}(\Omega)$ is a weak solution of the above problem and let $u_{h} \in V_{h}$ denote its Galerkin approximations over uniform triangulations, i.e., when two adjacent triangles form a parallelogram. Then for a strongly regular family of uniform triangulations we have (see [1], p. 498)

$$
\left\|u_{h}-L_{h} u\right\|_{1} \leq C h^{2}\|u\|_{3} \quad \text { as } h \rightarrow 0 .
$$

This phenomenon is called supercloseness (see [16]). It was first discovered by Oganesjan and Ruhovec [13].

Supercloseness has several important applications, e.g., in proving superconvergence of the finite element method for elliptic or parabolic problems (see $[11,13]$ ), or uniform convergence of finite element solution for singularly perturbed problems (see [5]). Superconvergence is also a useful tool in a posteriori error estimation, mesh refinement and adaptivity, higher order calculation of mechanical stresses or magnetic fields. In the next theorem we apply the above supercloseness property (3.3) to show that the ratio between the discretization error and the interpolation error behaves like $1+\mathcal{O}(h)$.

Theorem 3.1. Let $u \in H^{3}(\Omega) \cap C^{2+\varepsilon}(\bar{\Omega})$ for some $\varepsilon>0$. Then for a strongly regular family of uniform triangulations with $h \rightarrow 0$ we have

$$
\left(1-C_{1} h\right)\left|u-L_{h} u\right|_{1} \leq\left|u-u_{h}\right|_{1} \leq\left(1+C_{1} h\right)\left|u-L_{h} u\right|_{1}
$$

and

$$
\left(1-C_{2} h\right)\left\|u-L_{h} u\right\|_{1} \leq\left\|u-u_{h}\right\|_{1} \leq\left(1+C_{2} h\right)\left\|u-L_{h} u\right\|_{1} .
$$

Proof. If $u \in P_{1}(\Omega)$, then by (3.1) we have $u=L_{h} u=u_{h} \equiv 0$, and thus Theorem 3.1 holds. So let $u \notin P_{1}(\Omega)$. Now, according to the Poincaré-Friedrichs inequality, (3.3), and Theorem 2.2, there exists a constant $C_{3}>0$ such that

$$
C_{3}\left\|u_{h}-L_{h} u\right\|_{1} \leq\left|u_{h}-L_{h} u\right|_{1} \leq C_{4} h^{2}\|u\|_{3} \leq C_{1} h\left|u-L_{h} u\right|_{1},
$$

where $C_{1}>0$ depends on $u$. From this and the triangle inequality, we get the upper bounds of the discretization errors in (3.4) and (3.5),

$$
\left|u-u_{h}\right|_{1} \leq\left|u-L_{h} u\right|_{1}+\left|u_{h}-L_{h} u\right|_{1} \leq\left(1+C_{1} h\right)\left|u-L_{h} u\right|_{1}
$$

and

$$
\left\|u-u_{h}\right\|_{1} \leq\left\|u-L_{h} u\right\|_{1}+\left\|u_{h}-L_{h} u\right\|_{1} \leq\left(1+C_{2} h\right)\left\|u-L_{h} u\right\|_{1}
$$

By the triangle inequality and (3.6),

$$
\left|u-L_{h} u\right|_{1} \leq\left|u-u_{h}\right|_{1}+\left|u_{h}-L_{h} u\right|_{1} \leq\left|u-u_{h}\right|_{1}+C_{1} h\left|u-L_{h} u\right|_{1}
$$

for $h \rightarrow 0$, which implies the lower bound for the discretization error in (3.4).

Replacing the seminorms by norms in (3.7), we obtain the first inequality in (3.5). 
Remark 3.2. By the famous Céa's lemma there exists a constant $C$ such that

$$
\left\|u-u_{h}\right\| \leq C \inf _{v_{h} \in V_{h}}\left\|u-v_{h}\right\|
$$

where $\|\cdot\|$ is a norm in $V=H_{0}^{1}(\Omega)$. The right-hand side of (3.8) can be clearly estimated from above by $C\left\|u-u_{h}\right\|$. From this we find that the maximal possible lower bound is $1 \leq C$. This bound is attainable in an appropriate norm as we shall see from Theorem 3.3 below.

Céa's lemma plays an important role in finite element theory, since it enables us to transform the question of convergence of the finite element method (and a priori estimation for the discretization error) to the investigation of approximation properties of finite element spaces that are used. However, the constant $C$ coming from the standard proof of (3.8) can be very large (see [4]) especially when $\|\cdot\|=\|\cdot\|_{1}$ and $\mathcal{A}=\mathcal{A}(x)$ varies widely (e.g., if this coefficient is highly oscillating). We usually bound the right-hand side of (3.8) by the interpolation error, i.e.,

$$
\left\|u-u_{h}\right\|_{1} \leq C \inf _{v_{h} \in V_{h}}\left\|u-v_{h}\right\|_{1} \leq C\left\|u-L_{h} u\right\|_{1} .
$$

A similar relation is valid also for the norm $\|\cdot\|=|\cdot|_{1}$. From (3.4) and (3.5) we see that the supercloseness reduces the constant $C$ appearing on the right-hand side of $(3.9)$ to $1+\mathcal{O}(h)$ as $h \rightarrow 0$. The knowledge of the best possible value of $C$ is important in obtaining reliable a priori bounds for the discretization error.

For $v, w \in V$ denote by $a(v, w)=(\mathcal{A} \nabla v, \nabla w)_{0}$ the bilinear form corresponding to (3.1) and let

$$
\|v\|=\sqrt{a(v, v)}
$$

be the standard energy norm, which is equivalent to the $\|\cdot\|_{1}$-norm. The two-sided error estimate in the next theorem shows that the ratio between the discretization error and the interpolation error in norm (3.10) can even be much better than $1+\mathcal{O}(h)$. Note that the knowledge of constants in finite element analysis is very important from practical point of view.

Theorem 3.3. Let $u \in H^{3}(\Omega) \cap C^{2+\varepsilon}(\bar{\Omega})$ for some $\varepsilon>0$. Then for a strongly regular family of uniform triangulations with $h \rightarrow 0$ we have

$$
\left(1-C h^{2}\right)\left\|u-L_{h} u\right\| \leq\left\|u-u_{h}\right\| \leq\left\|u-L_{h} u\right\|
$$

Proof. Using the orthogonality relation $a\left(u-u_{h}, v_{h}\right)=0$ for all $v_{h} \in V_{h}$ and the symmetry of $a(.,$.$) , we get$

$$
\begin{aligned}
\left\|u-u_{h}\right\|^{2}+\left\|u_{h}-L_{h} u\right\|^{2} & =a\left(u-u_{h}, u-u_{h}\right)+2 a\left(u-u_{h}, u_{h}-L_{h} u\right)+a\left(u_{h}-L_{h} u, u_{h}-L_{h} u\right) \\
& =a\left(u-u_{h}, u-L_{h} u\right)+a\left(u-L_{h} u, u_{h}-L_{h} u\right) \\
& =a\left(u-u_{h}+u_{h}-L_{h} u, u-L_{h} u\right)=\left\|u-L_{h} u\right\|^{2}
\end{aligned}
$$

i.e., the right-hand inequality in (3.11) holds without any assumption on the partition of $\Omega$.

If $u \in P_{1}(\Omega)$, then by (3.1) we have $u=L_{h} u=u_{h} \equiv 0$, and thus Theorem 3.3 holds. So let $u \notin P_{1}(\Omega)$. From (3.12), (3.3) which holds for uniform partitions, and Theorem 2.2 we see that there exist constants $C_{1}, C>0$ such that

$$
1 \geq \frac{\left\|u-u_{h}\right\|}{\left\|u-L_{h} u\right\|}=\sqrt{1-\frac{\left\|u_{h}-L_{h} u\right\|^{2}}{\left\|u-L_{h} u\right\|^{2}}} \geq \sqrt{1-\frac{C_{1} h^{4}}{\left\|u-L_{h} u\right\|^{2}}} \geq \sqrt{1-C h^{2}}
$$

for $h \rightarrow 0$. Using the Taylor expansion $\sqrt{1-C h^{2}}=1-\frac{C h^{2}}{2}+\mathcal{O}\left(h^{4}\right)$, we find that

$$
\left(1-C h^{2}\right)\left\|u-L_{h} u\right\| \leq\left\|u-u_{h}\right\| \text { as } h \rightarrow 0 .
$$


Remark 3.4. According to [17], the upper bound of order $\mathcal{O}\left(h^{2}\right)$ in $(3.3)$ can be weakened to $\mathcal{O}\left(h^{1+\theta}\right), \theta \in(0,1]$, for weakly uniform triangulations, i.e., when two adjacent triangles form almost a parallelogram. Theorems 3.1 and 3.3 can be then correspondingly modified.

\section{BilineAR ELEMENTS}

Let $B_{h}$ be the standard bilinear Lagrangian interpolation operator on the square mesh defined for continuous functions $v$, i.e., $B_{h} v$ is also continuous and $\left.B_{h} v\right|_{S} \in Q_{1}(S)$ is a bilinear function on each square $S$ of the mesh.

Theorem 4.1. Let $v \in C^{2+\varepsilon}(\bar{\Omega}) \backslash Q_{1}(\bar{\Omega})$ for some $\varepsilon>0$. Then for a family of square meshes there exists a constant $C>0$ depending on $v$ such that

$$
\left|v-B_{h} v\right|_{1} \geq C h \quad \text { as } h \rightarrow 0 .
$$

Proof. Since $v$ is not bilinear, it follows that

$$
M:=\max _{(x, y) \in \bar{\Omega}}\left(\left(\partial_{11} v(x, y)\right)^{2}+\left(\partial_{22} v(x, y)\right)^{2}\right)>0 .
$$

Thus, there exists a nonempty subdomain $\Omega_{0} \subset \Omega$ such that for any $(x, y) \in \Omega_{0}$ we have

$$
\left(\partial_{11} v(x, y)\right)^{2}+\left(\partial_{22} v(x, y)\right)^{2}>\frac{M}{2} .
$$

Let $S \subset \Omega_{0}$ be a square with vertices $\left(x_{i}, y_{j}\right),\left(x_{i}+h, y_{j}\right),\left(x_{i}+h, y_{j}+h\right)$, and $\left(x_{i}, y_{j}+h\right)$. First, we again compute $\left|v-B_{h} v\right|_{1, S}^{2}$ under the assumption that $v$ is quadratic, i.e., $\partial_{11} v=a, \partial_{12} v=b$, and $\partial_{22} v=c$ are constants. In this special case we obtain

$$
\begin{aligned}
& \partial_{1}\left(v-B_{h} v\right)(x, y)=a\left(x-x_{i}-\frac{1}{2} h\right), \\
& \partial_{2}\left(v-B_{h} v\right)(x, y)=c\left(y-y_{j}-\frac{1}{2} h\right) .
\end{aligned}
$$

To see this, it is enough to verify (4.3) for two purely quadratic terms $\frac{a}{2} x^{2}$ and $\frac{c}{2} y^{2}$.

From (4.3) we immediately see that

$$
\begin{aligned}
\left|v-B_{h} v\right|_{1, S}^{2} & =\int_{S}\left(\left(\partial_{1}\left(v-L_{h} v\right)\right)^{2}+\left(\partial_{2}\left(v-L_{h} v\right)\right)^{2}\right) \mathrm{d} S \\
& =a^{2} h \int_{x_{i}}^{x_{i}+h}\left(x-x_{i}-\frac{1}{2} h\right)^{2} \mathrm{~d} x+c^{2} h \int_{y_{j}}^{y_{j}+h}\left(y-y_{j}-\frac{1}{2} h\right)^{2} \mathrm{~d} y \\
& =\frac{h^{4}}{12}\left(a^{2}+c^{2}\right) .
\end{aligned}
$$

This value is positive due to (4.2). From this we get similarly to (2.7) and (2.8) the lower bound (4.1).

The following supercloseness for bilinear elements can be found, e.g., in [6], [10], p. 314, [18], p. 9,

$$
\left\|u_{h}-B_{h} u\right\|_{1} \leq C h^{2}\|u\|_{3} \quad \text { as } h \rightarrow 0,
$$

where $u_{h}$ is a continuous piecewise bilinear finite element solution of (3.1). Now the proof of Theorems 3.1 and 3.3 for bilinear elements is essentially the same as for linear elements.

For a strongly regular family of uniform rectangular (nonsquare) meshes formulae (4.1) and (4.4) hold as well. The corresponding analysis is thus similar. 
TABLE 1. Numerical results for Example 5.1.

\begin{tabular}{|r|c|c|c|c|c|}
\hline$h^{-1}$ & $\left|u-u_{h}\right|_{1}$ & $\left|u-L_{h} u\right|_{1}$ & $\left|u_{h}-L_{h} u\right|_{1}$ & $1-C_{h}$ & $C_{h}$ \\
\hline 4 & 0.058775737 & 0.059199680 & 0.007072123 & 0.007161247 & 0.992838753 \\
\hline 8 & 0.030161134 & 0.030221195 & 0.001904372 & 0.001987383 & 0.998012617 \\
\hline 16 & 0.015180770 & 0.015188520 & 0.000485122 & 0.000510212 & 0.999489788 \\
\hline 32 & 0.007603031 & 0.007604008 & 0.000121857 & 0.000128412 & 0.999871588 \\
\hline
\end{tabular}

TABLE 2. Numerical results for Example 5.1.

\begin{tabular}{|r|c|c|c|c|c|}
\hline$h^{-1}$ & $\left\|u-u_{h}\right\|_{1}$ & $\left\|u-L_{h} u\right\|_{1}$ & $\left\|u_{h}-L_{h} u\right\|_{1}$ & $1-C_{h}$ & $C_{h}$ \\
\hline 4 & 0.059027858 & 0.059353209 & 0.007222771 & 0.005481610 & 0.994518390 \\
\hline 8 & 0.030195558 & 0.030241036 & 0.001948652 & 0.001503846 & 0.998496154 \\
\hline 16 & 0.015185172 & 0.015191020 & 0.000496651 & 0.000385017 & 0.999614983 \\
\hline 32 & 0.007603585 & 0.007604321 & 0.000124768 & 0.000096838 & 0.999903162 \\
\hline
\end{tabular}

TABLE 3. Numerical results for Example 5.2.

\begin{tabular}{|r|c|c|c|c|c|}
\hline$h^{-1}$ & $\left|u-u_{h}\right|_{1}$ & $\left|u-L_{h} u\right|_{1}$ & $\left|u_{h}-L_{h} u\right|_{1}$ & $1-C_{h}$ & $C_{h}$ \\
\hline 4 & 0.060204808 & 0.059202235 & 0.010173002 & -0.01693472 & 1.01693472 \\
\hline 8 & 0.030588994 & 0.030222536 & 0.004332142 & -0.01212533 & 1.01212533 \\
\hline 16 & 0.015253591 & 0.015189213 & 0.001297402 & -0.00423840 & 1.00423840 \\
\hline 32 & 0.007612842 & 0.007604386 & 0.000334686 & -0.00111207 & 1.00111207 \\
\hline 64 & 0.003804323 & 0.003803473 & 0.000077143 & -0.00022355 & 1.00022355 \\
\hline
\end{tabular}

\section{NUMERICAL EXPERIMENTS}

In the next two examples we use triangulations from Theorem 2.1.

Example 5.1. Consider the Poisson equation $-\Delta u=f$ in $\Omega=(0,1) \times(0,1)$ with homogeneous Dirichlet boundary conditions. The exact solution is chosen as follows: $u(x, y)=\left(x-x^{2}\right)\left(y-y^{2}\right)$. Then the corresponding right-hand side is given by $f(x, y)=2\left(x-x^{2}+y-y^{2}\right)$. All integrals were calculated by the quadrature formula from [8], p. 58, which is exact for all quintic polynomials on triangles. For numerical results see Tables 1 and 2, where $C_{h}=\left|u-u_{h}\right|_{1} /\left|u-L_{h} u\right|_{1}$ and $C_{h}=\left\|u-u_{h}\right\|_{1} /\left\|u-L_{h} u\right\|_{1}$, respectively. The last column of Table 2 shows that the constant $C$ appearing in (3.9) can be reduced to $1+\mathcal{O}(h)$.

Example 5.2. Let again $\Omega=(0,1) \times(0,1)$ and let

$$
\mathcal{A}(x, y)=\left(\begin{array}{cc}
100 x^{2}+1 & 0 \\
0 & 100 y^{2}+1
\end{array}\right)
$$

which clearly satisfies (3.2). The exact solution is chosen as in the previous example. Then the corresponding right-hand side of (3.1) is given by $f(x, y)=-1200 x^{2} y^{2}+800 x^{2} y+800 x y^{2}-400 x y-2 x^{2}-2 y^{2}+2 x+2 x$. In Tables 3 and 4 we see similar results as those in Example 5.1.

From the last columns we observe that the ratio between the discretization error and the interpolation error in the $H^{1}$-seminorm and norm seems to be even $1+\mathcal{O}\left(h^{2}\right)$, i.e., better than stated in Theorem 3.1.

According to [7], p. 246, the minimal possible constant appearing in the Friedrichs inequality $\|w\|_{0} \leq C_{0}|w|_{1}$ for all $w \in H_{0}^{1}(\Omega)$ is $C_{0}=2^{-1 / 2} / \pi$. Hence, $\|u\|_{1}^{2} \leq\left(1+1 /\left(2 \pi^{2}\right)\right)|u|_{1}^{2}$. The maximal and minimal eigenvalues of the matrix $\mathcal{A}(x, y)$ from $(5.1)$ equal to 101 and 1 , respectively. They are attained at the vertices $(1,1)$ and $(0,0)$, 
TABLE 4. Numerical results for Example 5.2.

\begin{tabular}{|r|c|c|c|c|c|}
\hline$h^{-1}$ & $\left\|u-u_{h}\right\|_{1}$ & $\left\|u-L_{h} u\right\|_{1}$ & $\left\|u_{h}-L_{h} u\right\|_{1}$ & $1-C_{h}$ & $C_{h}$ \\
\hline 4 & 0.060357589 & 0.059355945 & 0.010240942 & -0.01687521 & 1.01687521 \\
\hline 8 & 0.030608735 & 0.030242479 & 0.004361628 & -0.01211065 & 1.01211065 \\
\hline 16 & 0.015256190 & 0.015191767 & 0.001305424 & -0.00424065 & 1.00424065 \\
\hline 32 & 0.007613208 & 0.007604728 & 0.000336609 & -0.00111503 & 1.00111503 \\
\hline 64 & 0.003804395 & 0.003803532 & 0.000077551 & -0.00022699 & 1.00022699 \\
\hline
\end{tabular}

respectively. The above facts imply that the standard choice of the constant $C$ appearing in Céa's lemma (see [4], p. 105) is about $C=106.1$ which is much larger than the constants $C_{h}$.

Acknowledgements. The authors are indebted to Jan Brandts for fruitful discussions. This research was supported by Grant no. IAA 100190803 of the Grant Agency of the Academy of Sciences of the Czech Republic and the Chinese 973 Project under Grant number 2007CB814906, and Natural Science of Shandong Province of China under Grant number Y2008A18.

\section{REFERENCES}

[1] J. Brandts and M. Křížek, Gradient superconvergence on uniform simplicial partitions of polytopes. IMA J. Numer. Anal. 23 (2003) 489-505.

[2] W. Chen and M. Křížek, What is the smallest possible constant in Céa's lemma? Appl. Math. 51 (2006) 128-144.

[3] W. Chen and M. Kř́žzek, Lower bounds for the interpolation error for finite elements. Mathematics in Practice and Theory 39 (2009) 159-164 (in Chinese).

[4] P.G. Ciarlet, The finite element method for elliptic problems. North-Holland, Amsterdam (1978).

[5] S. Franz and T. Linss, Superconvergence analysis of the Galerkin FEM for a singularly perturbed convection-diffusion problems with characteristic layers. Numer. Methods Partial Differ. Equ. 24 (2008) 144-164.

[6] Ch. Grossmann, H.-G. Roos and M. Stynes, Numerical treatment of partial differential equations. Springer-Verlag, Berlin, Heidelberg (2007)

[7] S. Korotov, Two-sided a posteriori error estimates for linear elliptic problems with mixed boundary conditions. Appl. Math. 52 (2007) 235-249.

[8] M. Křížek and P. Neittaanmäki, Finite element approximation of variational problems and applications. Longman Scientific \& Technical, Harlow (1990).

[9] M. Kř́̌žek and P. Neittaanmäki, Mathematical and Numerical Modelling in Electrical Engineering: Theory and Applications. Kluwer, Dordrecht (1996).

[10] Q. Lin and J. Lin, Finite element methods: Accuracy and improvement. Science Press, Beijing (2006).

[11] G.I. Marchuk and V.I. Agoshkov, Introduction aux méthodes des éléments finis. Mir, Moscow (1985).

[12] J. Nečas and I. Hlaváček, Mathematical theory of elastic and elasto-plastic bodies: An introduction. Elsevier, Amsterdam (1981)

[13] L.A. Oganesjan and L.A. Ruhovec, An investigation of the rate of convergence of variational-difference schemes for second order elliptic equations in a two-dimensional region with smooth boundary. Ž. Vyčisl. Mat. i Mat. Fyz. 9 (1969) 1102-1120.

[14] G. Strang and G. Fix, An analysis of the finite element method. Prentice-Hall, Inc., Englewood Cliffs, New Jersey (1973).

[15] R. Verfürth, A review of a posteriori error estimation and adaptive mesh-refinement techniques. John Wiley \& Sons, Chichester, Teubner, Stuttgart (1996).

[16] L.B. Wahlbin, Superconvergence in Galerkin finite element methods, Lect. Notes in Math. 1605. Springer, Berlin (1995).

[17] L. Xu and Z. Zhang, Analysis of recovery type a posteriori error estimation for mildly structured grids. Math. Comp. 73 (2004) $1139-1152$.

[18] N.N. Yan, Superconvergence analysis and a posteriori error estimation in finite element methods. Science Press, Beijing (2008). 